\title{
The True Story on Deficiencies After Sleeve Gastrectomy: Results of a Double-Blind RCT
}

\author{
Laura Heusschen $^{1,2}$ (D) Wendy Schijns ${ }^{1} \cdot$ Nadine Ploeger $^{1} \cdot$ Laura N. Deden $^{1} \cdot$ Eric J. Hazebroek ${ }^{1} \cdot$ Frits J. Berends $^{1} \cdot$ \\ Edo O. Aarts ${ }^{1}$
}

Published online: 27 November 2019

(C) The Author(s) 2019

\begin{abstract}
Background Since a few years, the laparoscopic sleeve gastrectomy (SG) has become the most performed bariatric operation worldwide. However, as with all bariatric procedures, SG also leads to vitamin and mineral deficiencies post-operatively and standard multivitamin supplements are probably not sufficient.

Objective The present study evaluates the effectiveness of a specialized multivitamin supplement for SG patients (WLS Optimum 1.0, FitForMe, Rotterdam, the Netherlands), compared to a standard multivitamin supplement (sMVS).

Design A double-blind randomized controlled trial was performed. For 12 months, patients in the intervention group received WLS Optimum, containing elevated doses of multiple vitamins and minerals. Patients in the control group were provided with sMVS, containing $100 \%$ of the recommended dietary allowance.

Results In total, 139 patients were available for analysis (WLS Optimum, $n=69$; sMVS, $n=70$ ). Intention-to-treat analyses revealed more folic acid deficiencies and higher serum vitamin B1 levels in the WLS Optimum group. Per protocol analyses showed that in patients using WLS Optimum, serum folic acid and vitamin B1 levels were higher, serum PTH levels were lower, and only one patient $(2.6 \%)$ was anemic compared to 11 patients $(17.5 \%)$ using a sMVS $(p<0.05$ for all). No differences were found in prevalence of deficiencies for iron, vitamin B12, vitamin D, and other vitamins and minerals.

Conclusions This optimized multivitamin supplement only affected serum levels of folic acid, PTH and vitamin B1, and anemia rates compared to a sMVS. There is a clear need to further optimize multivitamin supplementation for SG patients. Besides, noncompliance with multivitamin supplements remains an important issue that should be dealt with.

Clinical Trial Registry The study protocol was registered at the clinical trials registry of the National Institutes of Health (ClinicalTrials.gov; identifier NCT01609387).
\end{abstract}

Keywords Morbid obesity $\cdot$ Bariatric surgery $\cdot$ Sleeve gastrectomy $\cdot \mathrm{SG} \cdot$ Deficiencies, micronutrients, vitamins, minerals

\begin{tabular}{|c|c|}
\hline \multicolumn{2}{|c|}{ Abbreviations } \\
\hline EWL & Excess weight loss \\
\hline LSG & laparoscopic sleeve gastrectomy \\
\hline $\mathrm{MCV}$ & mean corpuscular volume \\
\hline MVS & multivitamin supplements \\
\hline PPI & proton-pump inhibitors \\
\hline PTH & parathyroid hormone \\
\hline
\end{tabular}

Laura Heusschen

LHeusschen@Rijnstate.nl

1 Department of Surgery, Vitalys Clinic and Rijnstate Hospital, Wagnerlaan 55, Arnhem 6800 TA, The Netherlands

2 Divison of Human Nutrition and Health, Wageningen University, Wageningen, The Netherlands
RDA recommended dietary allowance

RHA Rijnstate Hospital Arnhem

RYGB Roux-en-Y gastric bypass

SG Sleeve gastrectomy

sMVS standard multivitamin supplements

TWL total weight loss

WE Wernicke's encephalopathy

\section{Introduction}

Originally designed as the first step of a biliopancreatic diversion with duodenal switch, the sleeve forming gastrectomy (SG) was technically improved and implemented as a standalone procedure [1]. Since a few years, the 
laparoscopic SG has become the most performed bariatric operation worldwide [2]. It is considered to be an easy, quick, and safe procedure [3] that provides significant weight loss and improvement of obesity-related comorbidities by reducing food intake and hormonal changes [3, 4].

Perhaps one of the reasons why the Roux-en-Y gastric bypass (RYGB) is no longer the preferred procedure for many surgeons is because it is associated with vitamin and mineral deficiencies and lifelong use of supplements $[5,6]$. Since the anatomy of the intestinal tract remains unaltered when performing an SG, the risk of developing deficiencies is theoretically considered lower [7]. Some authors even state that an SG has minimal impact on nutrient status [3] and taking multivitamin supplements (MVS) for more than 3 months postoperatively is unnecessary [8]. However, short and midterm studies found that in SG patients, deficiencies are as common as in RYGB patients [9-12]. Especially deficiencies for iron, folic acid, vitamin B12, and vitamin D are frequently reported [7, 13-17]. Standard MVS are probably not sufficient to prevent nutritional deficiencies after SG. However, specific MVS, which contain higher doses of vitamins and minerals, were not available at the time of this study. Based on literature and studies performed in our hospital, a customized MVS for SG patients was developed in 2012 (WLS Optimum; FitForMe, Rotterdam, the Netherlands). The present study evaluates the effectiveness of this SG-specific MVS compared to a standard MVS in a double-blind randomized controlled trial.

\section{Subjects and Methods}

\section{Study Design}

The present study was a double-blind randomized controlled trial. All patients who underwent a primary laparoscopic SG (LSG) operation at Rijnstate Hospital Arnhem (RHA; >1200 bariatric cases a year) between November 2011 and October 2014 were eligible for the study. Exclusion criteria were a secondary LSG, creatinine > $150 \mu \mathrm{mol} / \mathrm{L}$, liver enzymes $>2$ times the upper limit, concomitant diseases (e.g., gastrointestinal diseases), psychiatric illness, use of drugs that affect bone metabolism, and known pregnancy. The study was approved by the Medical Ethics Review Committee of Radboud University Medical Centre and the Local Ethical Committee of RHA, and was conducted in concordance with the principles of the Declaration of Helsinki. The study protocol was registered at the clinical trials registry of the National Institutes of Health (ClinicalTrials.gov; identifier NCT01609387).

Included patients were randomized into two groups: the intervention group received the customized MVS for SG patients (WLS Optimum) and the control group received a standard MVS (sMVS).

\section{Surgical Procedure}

A standardized operating technique was performed by three experienced bariatric surgeons ( $>500$ cases each). First, the greater omentum was dissected from the greater curvature of the stomach using Enseal ${ }^{\circledR}$ (Ethicon, Somerville USA). Then, the stomach and angle of His were mobilized, using a posterior approach. This was completed by dissection of the anterior part of the angle of His and small gastric vessels. Next, transection of the stomach was performed using lengthwise stapling along a 40 French calibration bougie positioned along the lesser curvature, starting $4 \mathrm{~cm}$ proximal of the pylorus until the cardia (Echelon Flex ${ }^{\mathrm{TM}}$ Powered Plus Stapler, Ethicon, Somerville USA). A bougie size of $40 \mathrm{Fr}$ is associated with a significant lower leak rate and similar weight loss results compared to smaller bougie sizes $[18,19]$. The remnant of the stomach was retrieved through an enlarged port incision in the left flank. This port was closed with Vicryl (Ethicon, Somerville USA) using a suture retriever. Finally, the skin was closed with agraves.

\section{Intervention and Control}

WLS Optimum version 1.0 is a customized MVS for SG patients and contains elevated doses of multiple vitamins and minerals (Table 1). A sMVS, similar to an over the counter MVS, served as a control and contained most micronutrients in a dose equivalent to $100 \%$ of the RDA.

To prevent bias, both supplements had the exact same raw base compounds and cherry flavor and were similar in color and size (Fig. 1). Both supplements were dosed as one capsule per day.

\section{Additional Medication}

All patients received fraxiparin (nadroparin, $0.6 \mathrm{mg} / 5700 \mathrm{IU}$ daily) for 6 weeks and proton-pump inhibitor (omeprazole, $20 \mathrm{mg}$ daily) for 6 months, as part of the standard postoperative protocol. All patients were additionally prescribed calcium/cholecalciferol 500/800 three times a day.

\section{Randomization and Blinding}

The allocation sequence was computer generated, using a variable block schedule. Besides an independent pharmacist, no one had access to the randomization list to ensure allocation concealment. All supplements were packaged in nonmarked blisters with the same expiration date, each containing 12 capsules. The blisters were packaged in a nonmarked sealed box and numbered according to the randomization list. After the last visit of the last study patient, the unblinded randomization list was available to the research team. No earlier unblinding occurred. 
Table 1 Content of intervention and control supplement

\begin{tabular}{|c|c|c|c|c|}
\hline \multirow[b]{2}{*}{ Ingredients } & \multicolumn{2}{|l|}{ sMVS } & \multicolumn{2}{|c|}{ WLS Optimum } \\
\hline & Dose & RDA (\%) & Dose & RDA (\%) \\
\hline \multicolumn{5}{|l|}{ Vitamins } \\
\hline Vitamin A, mg & 0.60 & 75.0 & 1.00 & 125.0 \\
\hline Vitamin B1, mg & 1.10 & 99.7 & 2.00 & 182.0 \\
\hline Vitamin B2, mg & 1.40 & 100.0 & 2.00 & 143.0 \\
\hline Vitamin B3, mg & 16.00 & 100.0 & 25.00 & 156.0 \\
\hline Vitamin B5, mg & 6.00 & 100.0 & 9.00 & 150.0 \\
\hline Vitamin B6, mg & 1.40 & 100.2 & 2.00 & 143.0 \\
\hline Biotin, $\mu \mathrm{g}$ & 25.00 & 50.0 & 150.00 & 300.0 \\
\hline Folic acid, $\mu \mathrm{g}$ & 200.00 & 100.0 & 300.00 & 150.0 \\
\hline Vitamin B12, $\mu \mathrm{g}$ & 2.50 & 100.0 & 10.00 & 400.0 \\
\hline Vitamin $\mathrm{C}, \mathrm{mg}$ & 80.00 & 100.0 & 100.00 & 125.0 \\
\hline Vitamin D, $\mu \mathrm{g}$ & 4.00 & 80.0 & 7.50 & 150.0 \\
\hline Vitamin E, mg & 10.00 & 83.4 & 12.00 & 100.0 \\
\hline Vitamin $\mathrm{K} 1, \mu \mathrm{g}$ & 25.00 & 33.3 & 90.00 & 120.0 \\
\hline \multicolumn{5}{|l|}{ Minerals } \\
\hline Chrome, $\mu \mathrm{g}$ & 40.00 & 100.0 & 40.00 & 100.0 \\
\hline Iron, mg & 14.00 & 100.0 & 21.00 & 150.0 \\
\hline Iodine, $\mu \mathrm{g}$ & 153.70 & 102.5 & 150.00 & 100.0 \\
\hline Copper, mg & 1.00 & 100.0 & 1.00 & 100.0 \\
\hline Chloride, mg & 0.14 & 0.0 & 0.00 & 0.0 \\
\hline Magnesium, mg & 30.00 & 8.0 & 30.00 & 8.0 \\
\hline Calcium, mg & 91.43 & 11.4 & 0.00 & 0.0 \\
\hline Manganese, mg & 2.00 & 100.1 & 3.00 & 150.0 \\
\hline Molybdenum, $\mu \mathrm{g}$ & 50.00 & 100.0 & 50.00 & 100.0 \\
\hline Selenium, $\mu \mathrm{g}$ & 55.00 & 100.0 & 55.00 & 100.0 \\
\hline Zinc, mg & 10.00 & 100.0 & 15.00 & 150.0 \\
\hline \multicolumn{5}{|l|}{ Other ingredients } \\
\hline Choline, mg & 0.00 & - & 10.00 & - \\
\hline
\end{tabular}

$s M V S$ standard multivitamin supplement, $R D A$ recommended daily allowance

\section{Data Collection, Follow-Up, and Outcome}

Standard laboratory blood tests were performed at baseline (T0) and 6 (T6) and 12 months (T12) after surgery. This included mean corpuscular volume (MCV), hemoglobin, iron, ferritin, folic acid, vitamin B12, vitamin D, PTH, calcium, magnesium, phosphate, albumin, vitamin B1, vitamin B6, and zinc (reference values in tables). Calcium levels were corrected for albumin using the following equation: $\mathrm{Ca}_{\text {corr }}=$ total calcium $-(0.025 \times$ albumin $)+1$.

Iron deficiency was the primary outcome measure. Secondary outcome measures included vitamin $\mathrm{D}$ and vitamin B12 deficiencies developed during the first 12 months after LSG.

Excess weight loss (EWL) was calculated as [weight loss/ excess weight based on ideal body weight at BMI $25 \mathrm{~kg} / \mathrm{m}^{2} \times$ $100 \%$ ]. Total body weight loss (TWL) was calculated as [weight loss/initial weight $\times 100 \%$ ].

\section{Correction of Deficiencies}

Preoperative vitamin B12 and vitamin D deficiencies were treated with predefined medication. If a deficiency occurred after surgery, it was recorded for the purpose of this study where after the deficiency was treated according to local protocol. After additional supplementation, subsequent data of the corresponding parameter was excluded to not bias mean serum level data. Moreover, follow-up measurements of patients who were pregnant at T6 and/or T12 were removed from the analyses.

\section{Sample Size Calculation and Statistical Analyses}

Sample size calculation was performed using Openepi.com [20]. To detect a $25 \%$ reduction of iron deficiency at 12 months after surgery, with 95\% sensitivity and a power of $90 \%$, a minimum of 56 patients per group was required. Taking into account a $10 \%$ dropout rate and $15 \%$ of cases excluded because of iron deficiency diagnosed and treated at 6 months, this resulted in 75 patients per group.
Fig. 1 Capsules + content of the standard multivitamin supplement (left) and WLS Optimum (right)

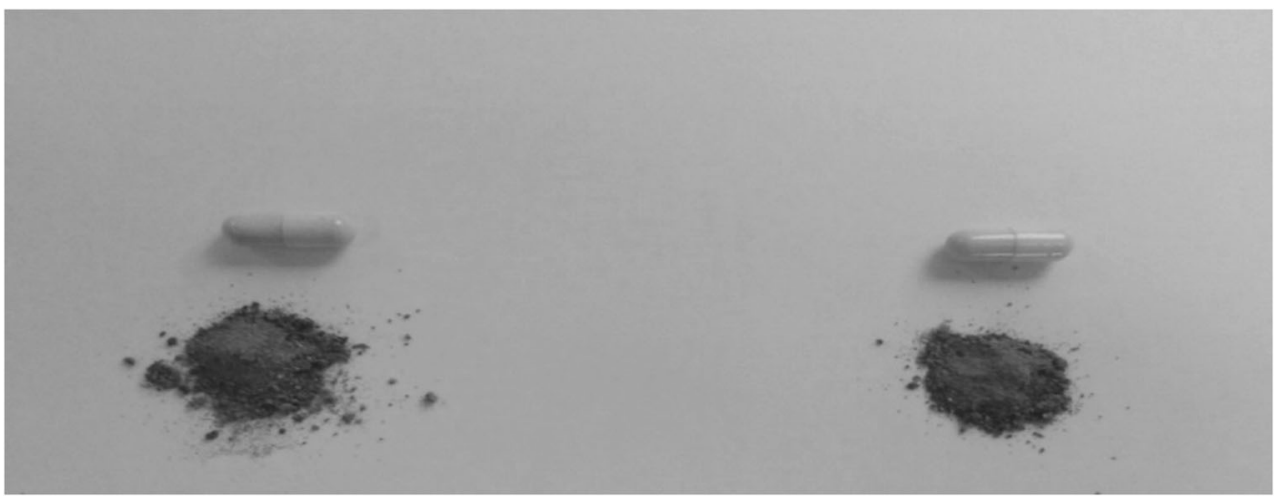


An intention-to-treat analysis was used as the primary analysis. Additionally, per protocol analyses were performed. Differences between groups at baseline, T6, and T12 were calculated using independent sample $t$ tests for continuous data and Chi-square tests for discrete data (or Fisher's exact test when $>20 \%$ of expected counts were $<5$ ). Linear mixed models were used to assess if serum levels changed differently over time between the groups.

Log transformations were performed to normalize the following data: serum levels of ferritin, $\mathrm{PTH}$, vitamin B1, and vitamin B6.

All statistical analyses were performed using IBM SPSS Statistics 25 for Windows (IBM Corp., Armonk USA).

\section{Results}

Eleven patients were excluded from analysis because they underwent a RYGB $(n=1)$, were pregnant during follow-up $(n=1)$, or did not complete any of the follow-up measurements $(n=9)$. In total, 139 patients were available for analysis: 69 patients receiving WLS Optimum and 70 patients receiving sMVS.

Both groups were similar at baseline with respect to age, gender, weight and BMI deficiencies (Table 2). The groups differed on the prevalence of dyslipidemia, which was three times higher in the sMVS group compared to the WLS Optimum group $(p=0.047)$.

In six patients (three in each group), a gastric band had to be removed before conversion to LSG ('Redo' surgery).

Table 2 Baseline characteristics of the study population

\begin{tabular}{lllll}
\hline & \multicolumn{2}{l}{ WLS Optimum $(n$ 69) } & \multicolumn{2}{c}{$\operatorname{sMVS}(n$ 70) } \\
\hline Age, year & 38.2 & \pm 12.4 & 39.7 & \pm 10.8 \\
Weight, kg & 141.3 & \pm 26.1 & 140.4 & \pm 31.2 \\
BMI, kg/m ${ }^{2}$ & 47.6 & \pm 9.0 & 48.4 & \pm 9.9 \\
Male & 18 & $(26.1)$ & 16 & $(22.9)$ \\
Redo surgery & 3 & $(4.3)$ & 3 & $(4.3)$ \\
Comorbidities & & & & \\
$\quad$ T2DM & 9 & $(13.0)$ & 7 & $(10.0)$ \\
$\quad$ Hypertension & 15 & $(21.7)$ & 19 & $(27.1)$ \\
$\quad$ Dyslipidemia & 3 & $(4.3)$ & 10 & $(14.3)$ \\
OSAS & 7 & $(10.1)$ & 7 & $(10.0)$ \\
\hline
\end{tabular}

Data are presented as mean \pm standard deviation and counts (percentages) MVS multivitamin supplement, OSAS obstructive sleep apnea syndrome, T2DM type 2 diabetes mellitus

$p>0.05$ for all

\section{Weight Loss}

The degree of weight loss after 12 months was similar in both groups. Mean BMI at T12 was $32.7 \mathrm{~kg} / \mathrm{m}^{2}$ in the WLS Optimum group and $33.8 \mathrm{~kg} / \mathrm{m}^{2}$ in the sMVS group. Furthermore, patients using WLS Optimum showed $70.5 \pm$ $22.7 \% \mathrm{EWL}$ and $31.3 \pm 8.6 \% \mathrm{TWL}$ compared to $68.5 \pm 23.2$ $\% \mathrm{EWL}$ and $30.5 \pm 8.4 \% \mathrm{TWL}$ for patients using sMVS $(p>0.05$ for all).

\section{Pre-operative Deficiencies}

The number of pre-operative deficiencies and mean serum levels at baseline were comparable between the groups (Tables 3, 4, and 5). Pre-operative deficiencies for vitamin D (76.1\%), phosphate $(34.1 \%)$, and albumin $(12.2 \%)$ were most prevalent.

\section{Post-operative Deficiencies}

Mean serum levels and prevalence of deficiencies regarding hemoglobin metabolism, calcium and vitamin D metabolism, and vitamin B1, B6, and zinc can be found in Tables 3, 4, and 5.

At T6, mean serum vitamin B1 levels were significantly higher in patients using WLS Optimum, compared to sMVS users: $148.0 \pm 27.6 \mathrm{nmol} / \mathrm{L}$ vs $134.8 \pm 24.8 \mathrm{nmol} / \mathrm{L}(p=$ 0.011). Mean serum levels for all other parameters were comparable between the groups at 6 and 12 months of follow-up.

During the entire study period, overall significantly more patients in the WLS Optimum group were deficient for folic acid: 10 patients $(14.5 \%)$ versus 2 patients $(2.9 \%)$ in the sMVS group $(p=0.016)$. No differences were found in prevalence of anemia, and deficiencies for iron, vitamin B12, vitamin $\mathrm{D}$, and other vitamins and minerals.

Elevated serum B1 and B6 levels were found in $11(18.0 \%)$ and 20 patients $(32.8 \%)$ using WLS Optimum and $5(7.9 \%)$ and 13 patients $(20.6 \%)$ using sMVS $(p>0.05)$. For PTH, elevated serum levels were more frequent in the sMVS group (11 patients, $15.7 \%$ ) compared to the WLS Optimum group (4 patients, $5.8 \%)$, but this was also not significant $(p=0.060)$.

\section{Compliance}

Of the 69 patients in the intervention group, only 44 patients (63.8\%) reported using the WLS Optimum supplement after 6 months. This number decreased to 38 patients $(55.1 \%)$ after 12 months. The main reported reason for discontinuation was nausea. Most patients switched to an over the counter MVS. Others did not tolerate any MVS and therefore stopped using multivitamin supplementation. Based on self-reported compliance, the total group of patients was redivided into WLS 
Table 3 Evaluation of hemoglobin metabolism

\begin{tabular}{|c|c|c|c|c|c|c|c|c|c|}
\hline \multirow[t]{2}{*}{ Parameter } & \multirow[t]{2}{*}{ Cases } & \multicolumn{4}{|c|}{ Mean serum levels } & \multicolumn{4}{|c|}{ Deficiencies } \\
\hline & & \multicolumn{2}{|c|}{ WLS Optimum } & \multicolumn{2}{|l|}{$s M V S$} & \multicolumn{2}{|c|}{ WLS Optimum } & \multicolumn{2}{|c|}{$s M V S$} \\
\hline \multicolumn{10}{|c|}{ Hemoglobin: F: 7.4-9.9 mmol/L, M: 8.4-10.8 mmol/L (US: F:11.9-16.0 g/L, M: $13.5-17.4 \mathrm{~g} / \mathrm{L})$} \\
\hline T0 & $138(69 / 69)$ & 8.7 & \pm 0.8 & 8.8 & \pm 0.7 & 4 & $(5.8)$ & 4 & $(5.8)$ \\
\hline T6 & $134(67 / 67)$ & 8.6 & \pm 0.8 & 8.7 & \pm 0.8 & 3 & $(4.5)$ & 6 & $(9.0)$ \\
\hline $\mathrm{T} 12$ & $130(66 / 64)$ & 8.4 & \pm 0.7 & 8.5 & \pm 0.7 & 7 & $(10.6)$ & 7 & (10.9) \\
\hline $\mathrm{T} 0-\mathrm{T} 12$ & $129(65 / 64)$ & -0.25 & \pm 0.61 & -0.28 & \pm 0.52 & & & & \\
\hline \multicolumn{10}{|c|}{ MCV: $80-100 \mathrm{fL}$} \\
\hline T0 & $136(68 / 68)$ & 88.7 & \pm 4.1 & 88.9 & \pm 4.7 & 1 & (1.5) & 2 & $(2.9)$ \\
\hline T6 & $134(67 / 67)$ & 90.5 & \pm 3.3 & 90.6 & \pm 4.8 & 0 & - & 1 & $(1.5)$ \\
\hline $\mathrm{T} 12$ & $130(66 / 64)$ & 91.8 & \pm 4.4 & 91.7 & \pm 4.7 & 0 & - & 1 & $(1.6)$ \\
\hline $\mathrm{T} 0-\mathrm{T} 12$ & $128(65 / 63)$ & +3.20 & \pm 4.16 & +2.68 & \pm 2.94 & & & & \\
\hline \multicolumn{10}{|c|}{ Iron: 9-31 $\mu \mathrm{mol} / \mathrm{L}(U S: 50.3-173.1 \mu \mathrm{g} / d L)$} \\
\hline $\mathrm{T} 0$ & $128(61 / 67)$ & 10.8 & \pm 4.4 & 11.0 & \pm 4.7 & 18 & $(29.5)$ & 24 & $(35.8)$ \\
\hline T6 & $105(48 / 57)$ & 13.7 & \pm 4.2 & 14.1 & \pm 5.2 & 7 & $(14.6)$ & 8 & $(14.0)$ \\
\hline $\mathrm{T} 12$ & $108(55 / 51)$ & 15.1 & \pm 5.3 & 16.1 & \pm 7.0 & 7 & $(12.7)$ & 3 & $(5.9)$ \\
\hline $\mathrm{T} 0-\mathrm{T} 12$ & $98(50 / 48)$ & +4.56 & \pm 5.64 & +4.90 & \pm 6.20 & & & & \\
\hline \multicolumn{10}{|c|}{ Ferritin:20-300 ng/mL } \\
\hline $\mathrm{T} 0$ & $139(69 / 70)$ & 127.6 & \pm 96.4 & 128.8 & \pm 97.7 & 2 & $(2.9)$ & 3 & $(4.3)$ \\
\hline T6 & $112(53 / 59)$ & 149.0 & \pm 114.0 & 133.1 & \pm 73.6 & 0 & - & 4 & $(6.8)$ \\
\hline $\mathrm{T} 12$ & $131(65 / 64)$ & 139.4 & \pm 104.7 & 129.1 & \pm 74.5 & 2 & $(3.1)$ & 3 & $(4.7)$ \\
\hline $\mathrm{T} 0-\mathrm{T} 12$ & $131(65 / 64)$ & +8.09 & \pm 55.36 & -2.95 & \pm 78.59 & & & & \\
\hline \multicolumn{10}{|c|}{ Folic acid: $9.1-36 \mathrm{nmol} / \mathrm{L}(U S: 4.0-15.9 \mathrm{ng} / \mathrm{mL}$ ) } \\
\hline T0 & $137(68 / 69)$ & 16.6 & \pm 6.7 & 16.7 & \pm 6.0 & 2 & $(2.9)$ & 4 & $(5.8)$ \\
\hline T6 & $132(66 / 66)$ & 22.3 & \pm 9.5 & 19.8 & \pm 6.9 & 5 & (7.6) & 0 & - \\
\hline $\mathrm{T} 12$ & $131(66 / 65)$ & 21.8 & \pm 10.0 & 19.2 & \pm 6.7 & 5 & $(7.6)$ & 2 & $(3.1)$ \\
\hline $\mathrm{T} 0-\mathrm{T} 12$ & $129(65 / 64)$ & +5.07 & \pm 9.16 & +2.72 & \pm 7.28 & & & & \\
\hline \multicolumn{10}{|c|}{ Vitamin B12: $145 / 200^{\mathrm{a}}-570 \mathrm{pmol} / \mathrm{L}\left(\right.$ US: $\left.197 / 271^{a}-773 \mathrm{pg} / \mathrm{mL}\right)$} \\
\hline T0 & $137(67 / 70)$ & 289.8 & \pm 96.4 & 315.9 & \pm 110.1 & 1 & $(1.5)$ & 0 & - \\
\hline T6 & $112(52 / 60)$ & 276.1 & \pm 84.6 & 291.7 & \pm 92.3 & 11 & (21.2) & 11 & (18.3) \\
\hline $\mathrm{T} 12$ & $122(59 / 63)$ & 267.3 & \pm 80.0 & 284.4 & \pm 85.7 & 15 & $(25.4)$ & 14 & $(22.2)$ \\
\hline T0-T12 & $122(59 / 63)$ & -32.93 & \pm 76.25 & -34.17 & \pm 91.11 & & & & \\
\hline
\end{tabular}

Data are presented as mean \pm standard deviation and counts (percentages)

${ }^{a}$ Normal range before the operation (baseline) was 145-570 pmol/L (US:197-773 pg/mL)

$F$ female, $M$ male, US United States (units), $M C V$ mean corpuscular volume, $s M V S$ standard multivitamin supplement

$p>0.05$ for all

Optimum users and sMVS-users. Results are shown in Table 6.

At T6 and T12, mean serum folic acid levels were significantly higher in patients using WLS Optimum, compared to patients using a sMVS: 24.1 and $24.4 \mathrm{mmol} / \mathrm{L}$ vs 20.2 and $19.6 \mathrm{mmol} / 1$ ( $p<0.05$ for both). This was also true for mean vitamin B1 levels at 6 months: $150.2 \pm 27.6 \mathrm{nmol} / \mathrm{L}$ for WLS optimum users and $137.9 \pm 23.3 \mathrm{nmol} / \mathrm{L}$ for sMVS users $(p=$ 0.028).

At 12 months, mean serum PTH levels were significantly lower in the group using WLS Optimum $(3.2 \pm 1.7 \mathrm{pmol} / \mathrm{L})$ compared to the group using a sMVS $(4.0 \pm 2.1)(p=0.026)$.
Throughout the entire study period, overall only one patient $(2.6 \%)$ using WLS Optimum was anemic compared to 11 of the patients $(17.5 \%)$ using a sMVS $(p=0.029)$. No significant differences were found for other vitamins and minerals.

\section{Discussion}

The present study demonstrated that the specialized multivitamin supplement WLS Optimum 1.0 had no clear advantages over standard supplementation as it was not associated with fewer micronutrient deficiencies after SG. 
Table 4 Evaluation of calcium and vitamin D metabolism

\begin{tabular}{|c|c|c|c|c|c|c|c|c|c|}
\hline \multirow[t]{2}{*}{ Parameter } & \multirow[t]{2}{*}{ Cases } & \multicolumn{4}{|c|}{ Mean serum levels } & \multicolumn{4}{|c|}{ Deficiencies } \\
\hline & & \multicolumn{2}{|c|}{ WLS Optimum } & \multicolumn{2}{|l|}{$s M V S$} & \multicolumn{2}{|c|}{ WLS Optimum } & \multicolumn{2}{|c|}{$s M V S$} \\
\hline \multicolumn{10}{|c|}{ Vitamin D: > $50 \mathrm{nmol} / \mathrm{L}(U S:>20 \mathrm{ng} / \mathrm{mL})$} \\
\hline T0 & $138(69 / 70)$ & 36.5 & \pm 21.8 & 34.0 & \pm 16.7 & 51 & $(73.9)$ & 54 & $(77.1)$ \\
\hline T6 & $133(66 / 67)$ & 86.7 & \pm 27.6 & 93.8 & \pm 36.8 & 5 & $(7.6)$ & 4 & $(6.0)$ \\
\hline $\mathrm{T} 12$ & $129(64 / 65)$ & 84.5 & \pm 32.3 & 89.7 & \pm 28.8 & 7 & $(10.9)$ & 4 & $(6.2)$ \\
\hline $\mathrm{T} 0-\mathrm{T} 12$ & $128(63 / 65)$ & +48.68 & \pm 29.21 & +55.62 & \pm 28.85 & & & & \\
\hline \multicolumn{10}{|c|}{ PTH: $1.3-6.8 \mathrm{pmol} / \mathrm{L}(U S: 12.3-64.1 \mathrm{pg} / \mathrm{mL})$} \\
\hline $\mathrm{T} 0$ & $139(69 / 70)$ & 3.7 & \pm 2.0 & 4.0 & \pm 2.5 & 1 & (1.4) & 4 & $(5.7)$ \\
\hline T6 & $134(66 / 68)$ & 3.4 & \pm 1.6 & 3.8 & \pm 1.9 & 2 & $(3.0)$ & 5 & $(7.4)$ \\
\hline $\mathrm{T} 12$ & $130(65 / 65)$ & 3.5 & \pm 2.0 & 4.0 & \pm 2.3 & 2 & (3.1) & 0 & - \\
\hline $\mathrm{T} 0-\mathrm{T} 12$ & $130(65 / 65)$ & -0.30 & \pm 2.10 & -0.09 & \pm 2.57 & & & & \\
\hline \multicolumn{10}{|c|}{ Calcium $^{\mathrm{a}}: 2.10-2.55 \mathrm{mmol} / \mathrm{L}$ (US: $8.42-10.22 \mathrm{mg} / \mathrm{dL}$ ) } \\
\hline T0 & $130(62 / 68)$ & 2.35 & \pm 0.11 & 2.35 & \pm 0.09 & 1 & (1.6) & 0 & - \\
\hline $\mathrm{T} 6$ & $133(65 / 68)$ & 2.40 & \pm 0.08 & 2.39 & \pm 0.08 & 0 & - & 0 & - \\
\hline $\mathrm{T} 12$ & $130(65 / 65)$ & 2.40 & \pm 0.08 & 2.38 & \pm 0.07 & 0 & - & 0 & - \\
\hline $\mathrm{T} 0-\mathrm{T} 12$ & $121(58 / 63)$ & +0.05 & \pm 0.11 & +0.03 & \pm 0.10 & & & & \\
\hline \multicolumn{10}{|c|}{ Magnesium: $0.70-1.10 \mathrm{mmol} / \mathrm{L}$ (US: $1.70-2.67 \mathrm{mg} / \mathrm{dL}$ ) } \\
\hline T0 & $130(63 / 67)$ & 0.80 & \pm 0.07 & 0.80 & \pm 0.06 & 3 & $(4.8)$ & 3 & $(4.5)$ \\
\hline T6 & $85(40 / 45)$ & 0.82 & \pm 0.05 & 0.83 & \pm 0.05 & 1 & $(2.5)$ & 0 & - \\
\hline $\mathrm{T} 12$ & $86(43 / 43)$ & 0.83 & \pm 0.06 & 0.83 & \pm 0.04 & 1 & $(2.3)$ & 0 & - \\
\hline $\mathrm{T} 0-\mathrm{T} 12$ & $80(40 / 40)$ & +0.03 & \pm 0.07 & +0.03 & \pm 0.06 & & & & \\
\hline \multicolumn{10}{|c|}{ Phosphate: $0.87-1.45 \mathrm{mmol} / \mathrm{L}$ (US: $2.70-4.50 \mathrm{mg} / \mathrm{dL}$ ) } \\
\hline $\mathrm{T} 0$ & $129(62 / 67)$ & 0.95 & \pm 0.18 & 0.93 & \pm 0.16 & 22 & $(35.5)$ & 22 & $(32.8)$ \\
\hline T6 & $106(49 / 57)$ & 1.01 & \pm 0.15 & 0.95 & \pm 0.15 & 10 & $(20.4)$ & 14 & $(24.6)$ \\
\hline $\mathrm{T} 12$ & $106(55 / 51)$ & 1.02 & \pm 0.20 & 1.02 & \pm 0.15 & 10 & $(18.2)$ & 7 & $(13.7)$ \\
\hline $\mathrm{T} 0-\mathrm{T} 12$ & $99(51 / 48)$ & +0.07 & \pm 0.20 & +0.07 & \pm 0.23 & & & & \\
\hline \multicolumn{10}{|c|}{ Albumin: $35-50 \mathrm{~g} / \mathrm{L}$} \\
\hline T0 & $131(63 / 68)$ & 37.8 & \pm 3.8 & 37.8 & \pm 2.6 & 9 & (14.3) & 7 & (10.3) \\
\hline T6 & $133(65 / 68)$ & 38.8 & \pm 3.4 & 39.0 & \pm 3.0 & 6 & $(9.2)$ & 5 & $(7.4)$ \\
\hline $\mathrm{T} 12$ & $133(65 / 65)$ & 38.5 & \pm 3.2 & 38.8 & \pm 2.7 & 8 & (12.3) & 3 & $(4.6)$ \\
\hline $\mathrm{T} 0-\mathrm{T} 12$ & $122(59 / 63)$ & +1.00 & \pm 3.50 & +1.03 & \pm 2.99 & & & & \\
\hline
\end{tabular}

Data are presented as mean \pm standard deviation and counts (percentages)

US United States (units), sMVS standard multivitamin supplement

${ }^{\mathrm{a}}$ Corrected for albumin levels (total calcium $-(0.025 \times$ albumin $\left.)+1\right)$

$p>0.05$ for all

Therefore, the content of this first version of WLS Optimum should be further optimized.

More importantly, the present study illustrates that nutritional deficiencies are highly prevalent after SG, despite the anatomy of the intestinal tract remaining unaltered. There are several factors that put patients at risk for developing nutritional deficiencies after SG, including reduced food intake, decreased hydrochloric acid and intrinsic factor secretion, vomiting, poor food choices, and food intolerance $[7,21]$. Yet, others believe that $\mathrm{SG}$ has minimal impact on nutrient status [3] and that maintenance of
MVS more than 3 months postoperatively seems to be of no benefit [8]. According to Ruiz-Tovar et al., once a patient is able to eat all kinds of food, additional vitamin and mineral supplementation can be discontinued [8]. In the present study, about three-quarters of the patients showed at least one micronutrient deficiency during the first year post-sleeve, despite the use of multivitamin supplements. In view of our findings, a specialized multivitamin supplement for SG patients should at least contain higher doses of elementary iron, vitamin B12, vitamin D, vitamin B1, and zinc to prevent nutritional deficiencies post-operatively. 
Table 5 Vitamin B1 and B6 and zinc

\begin{tabular}{|c|c|c|c|c|c|c|c|c|c|}
\hline \multirow[t]{2}{*}{ Parameter } & \multirow[t]{2}{*}{ Cases } & \multicolumn{4}{|c|}{ Mean serum levels } & \multicolumn{4}{|c|}{ Deficiencies } \\
\hline & & \multicolumn{2}{|c|}{ WLS Optimum } & \multicolumn{2}{|l|}{$s M V S$} & \multicolumn{2}{|c|}{ WLS Optimum } & \multicolumn{2}{|c|}{$s M V S$} \\
\hline \multicolumn{10}{|c|}{ Vitamin B1: 95-175 nmol/L (US: 3.2-5.9 $\mu \mathrm{g} / \mathrm{dL}$ ) } \\
\hline T0 & $128(61 / 67)$ & 167.8 & \pm 29.5 & 162.3 & \pm 31.1 & 0 & - & 1 & $(1.5)$ \\
\hline T6 & $104(49 / 55)$ & $148.0 *$ & \pm 27.6 & 134.8 & \pm 24.8 & 1 & $(2.0)$ & 4 & $(7.3)$ \\
\hline $\mathrm{T} 12$ & $106(54 / 52)$ & 145.4 & \pm 29.8 & 144.1 & \pm 44.1 & 2 & $(3.7)$ & 2 & $(3.8)$ \\
\hline $\mathrm{T} 0-\mathrm{T} 12$ & $99(50 / 49)$ & -19.35 & \pm 40.61 & -17.24 & \pm 45.05 & & & & \\
\hline \multicolumn{10}{|c|}{ Vitamin B6: $25-100 \mathrm{nmol} / \mathrm{L}$ (US: $0.62-2.47 \mu \mathrm{g} / \mathrm{dL}$ ) } \\
\hline $\mathrm{T} 0$ & $127(61 / 66)$ & 79.3 & \pm 24.0 & 75.3 & \pm 29.5 & 0 & - & 0 & - \\
\hline T6 & $104(49 / 55)$ & 91.7 & \pm 36.1 & 88.1 & \pm 63.0 & 0 & - & 0 & - \\
\hline $\mathrm{T} 12$ & $105(54 / 51)$ & 82.9 & \pm 27.3 & 78.2 & \pm 25.9 & 0 & - & 0 & - \\
\hline $\mathrm{T} 0-\mathrm{T} 12$ & $97(49 / 48)$ & +3.06 & \pm 26.59 & 5.85 & \pm 30.77 & & & & \\
\hline \multicolumn{10}{|c|}{$\operatorname{Zinc}(9.2-18.4 \mu \mathrm{mol} / \mathrm{L}(U S: 60-120 \mu \mathrm{g} / \mathrm{dL})$} \\
\hline $\mathrm{T} 0$ & $127(61 / 66)$ & 12.2 & \pm 1.6 & 12.2 & \pm 2.0 & 1 & (1.6) & 4 & (6.1) \\
\hline T6 & $105(48 / 57)$ & 11.7 & \pm 1.6 & 12.1 & \pm 1.9 & 3 & (6.3) & 2 & (3.5) \\
\hline $\mathrm{T} 12$ & $102(53 / 49)$ & 11.7 & \pm 1.9 & 11.8 & \pm 1.7 & 2 & (3.8) & 4 & $(8.2)$ \\
\hline T0-T12 & $95(50 / 45)$ & -0.44 & \pm 2.25 & -0.81 & \pm 2.15 & & & & \\
\hline
\end{tabular}

Data are presented as mean \pm standard deviation and counts (percentages)

US United States (units), sMVS standard multivitamin supplement

$* p<0.05$

\section{Anemia and Iron Deficiency}

In total, 17 patients (12.3\%) were anemic during the study. Additionally, three patients $(2.2 \%)$ had iron deficiency anemia. This is in line with the prospective cohort study of Hakeam et al., who also found a low prevalence of iron deficiency anemia (1.6\%) 1 year after SG [22]. In contrast, Abdulrahman and colleagues reported that $36 \%$ of their patients developed iron deficiency anemia [23]. However, the latter study most likely used serum iron concentrations to define iron deficiency anemia as they did not report on ferritin levels.

Post-bariatric anemia is in most cases due to iron deficiency, along with vitamin B12 deficiency as a secondary cause [5]. We observed iron deficiency, expressed in low serum ferritin levels, in seven patients $(5.1 \%)$. After surgery, reduced secretion of $\mathrm{HCl}$, use of proton-pump inhibitors (PPI), and faster gastric emptying may limit absorption [13, 14, 21, 24]. Besides low absorption, reduced oral intake and intolerance to iron-rich sources such as red meat might be a cause of iron deficiency post-sleeve $[14,21]$.

The dose of $21 \mathrm{mg}$ elementary iron in WLS Optimum 1.0 should be increased to prevent iron deficiencies post-sleeve. Yet, considering the low number of deficiencies observed in the present study, the recommended dose of 45-60 mg for WLS preventative supplements according to the ASMBS guidelines [25] is probably overestimated.

\section{Vitamin B12 Deficiency}

Occurrence of vitamin B12 deficiency after SG is mainly due to the reduction of $\mathrm{HCl}$ and intrinsic factor as a consequence of the surgery, which is even more pronounced with PPI intake $[14,26]$. In the present study, a marked decrease in mean serum vitamin B12 levels over the first year post-operatively was found in both groups, indicating that the dose of $10 \mu \mathrm{g}$ vitamin B12 in the WLS Optimum supplement was insufficient. In the study of Al-Mutawa and colleagues (2018), patients were prescribed additional B-complex tablets for 1-3 months, including $200 \mu \mathrm{g}$ vitamin B12 (next to $100 \mathrm{mg}$ vitamin B1 and $200 \mathrm{mg}$ vitamin B6) [14]. This high dose of vitamin B12 (8400\% RDA) significantly improved serum vitamin B12 levels during the early post-operative period in comparison to baseline. Thereafter, patients continued with a daily multivitamin supplement that provided only $1 \mu \mathrm{g}(42 \% \mathrm{RDA})$ of vitamin B12, which was insufficient to prevent deficiencies [14].

These findings indicate that SG-specific MVS do not need to contain more than $100 \mu \mathrm{g}$ of vitamin B12 to prevent deficiencies. This is not in line with the ASMBS recommendation of 350-500 $\mu \mathrm{g}$ per day [25], probably 
Table 6 Results of the per protocol analyses

\begin{tabular}{|c|c|c|c|c|c|c|c|c|c|}
\hline \multirow[t]{2}{*}{ Parameter } & \multirow[t]{2}{*}{ Cases } & \multicolumn{4}{|c|}{ Mean serum levels } & \multicolumn{4}{|c|}{ Deficiencies } \\
\hline & & \multicolumn{2}{|c|}{ WLS Optimum } & \multicolumn{2}{|l|}{$s M V S$} & \multicolumn{2}{|c|}{ WLS Optimum } & \multicolumn{2}{|c|}{$s M V S$} \\
\hline \multicolumn{10}{|c|}{ Hemoglobin: F: 7.4-9.9 mmol/L, M: 8.4-10.8 mmol/L (US: F:11.9-16.0 g/L, $M: 13.5-17.4 \mathrm{~g} / \mathrm{L}$ ) } \\
\hline T6 & $110(44 / 66)$ & 8.7 & \pm 0.7 & 8.7 & \pm 0.8 & 0 & - & 5 & $(8.2)$ \\
\hline $\mathrm{T} 12$ & $100(38 / 62)$ & 8.5 & \pm 0.6 & 8.5 & \pm 0.7 & 1 & $(2.6)$ & 8 & $(12.9)$ \\
\hline $\mathrm{T} 0-\mathrm{T} 12$ & $99(37 / 62)$ & -0.34 & \pm 0.66 & -0.20 & \pm 0.54 & & & & \\
\hline \multicolumn{10}{|c|}{ Ferritin:20-300 ng/mL } \\
\hline T6 & $93(37 / 56)$ & 148.3 & \pm 116.4 & 136.3 & \pm 74.2 & 0 & - & 2 & $(3.6)$ \\
\hline $\mathrm{T} 12$ & $99(37 / 62)$ & 150.0 & \pm 116.5 & 130.5 & \pm 75.9 & 1 & $(2.7)$ & 2 & $(3.2)$ \\
\hline $\mathrm{T} 0-\mathrm{T} 12$ & $99(37 / 62)$ & +2.65 & \pm 60.33 & -3.61 & \pm 80.05 & & & & \\
\hline \multicolumn{10}{|c|}{ Folic acid: $9.1-36 \mathrm{nmol} / \mathrm{L}(U S: 4.0-15.9 \mathrm{ng} / \mathrm{mL})$} \\
\hline T6 & $109(44 / 65)$ & $24.1 *$ & \pm 8.7 & 20.2 & \pm 7.0 & 0 & - & 0 & - \\
\hline $\mathrm{T} 12$ & $101(38 / 63)$ & $24.4 *$ & \pm 10.3 & 19.6 & \pm 6.6 & 1 & $(2.6)$ & 3 & $(4.8)$ \\
\hline $\mathrm{T} 0-\mathrm{T} 12$ & $100(37 / 63)$ & $+6.84 *$ & \pm 9.73 & +2.42 & \pm 7.05 & & & & \\
\hline \multicolumn{10}{|c|}{ Vitamin B12: 200-570 pmol/L (US: 271-773 pg/mL) } \\
\hline T6 & $93(36 / 57)$ & 278.9 & \pm 90.0 & 300.0 & \pm 88.8 & 8 & $(22.2)$ & 8 & $(14.0)$ \\
\hline $\mathrm{T} 12$ & $94(33 / 61)$ & 277.5 & \pm 77.8 & 286.0 & \pm 87.6 & 7 & $(21.2)$ & 14 & $(23.0)$ \\
\hline T0-T12 & $94(33 / 61)$ & -25.27 & \pm 83.19 & -29.30 & \pm 83.69 & & & & \\
\hline \multicolumn{10}{|c|}{ Vitamin D: > $50 \mathrm{nmol} / \mathrm{L}(U S:>20 \mathrm{ng} / \mathrm{mL})$} \\
\hline T6 & $110(44 / 66)$ & 87.4 & \pm 25.2 & 91.6 & \pm 32.8 & 1 & $(2.3)$ & 4 & $(6.1)$ \\
\hline $\mathrm{T} 12$ & $99(37 / 62)$ & 88.0 & \pm 28.4 & 86.9 & \pm 27.7 & 2 & $(5.4)$ & 5 & $(8.1)$ \\
\hline T0-T12 & $99(37 / 62)$ & +48.24 & \pm 28.22 & +53.77 & \pm 25.53 & & & & \\
\hline \multicolumn{10}{|c|}{ PTH: $1.3-6.8 \mathrm{pmol} / \mathrm{L}(U S: 12.3-64.1 \mathrm{pg} / \mathrm{mL})$} \\
\hline T6 & $111(44 / 67)$ & 3.1 & \pm 1.4 & 3.7 & \pm 1.7 & 2 & $(4.5)$ & 4 & $(6.0)$ \\
\hline $\mathrm{T} 12$ & $100(37 / 63)$ & $3.2 *$ & \pm 1.7 & 4.0 & \pm 2.1 & 1 & $(2.7$ & 0 & - \\
\hline $\mathrm{T} 0-\mathrm{T} 12$ & $100(37 / 63)$ & -0.32 & \pm 1.90 & -0.10 & \pm 2.66 & & & & \\
\hline \multicolumn{10}{|c|}{ Vitamin B1: 95-175 nmol/L (US: 3.2-5.9 $\mu \mathrm{g} / \mathrm{dL}$ ) } \\
\hline T6 & $86(36 / 50)$ & $150.2 *$ & \pm 27.6 & 137.9 & \pm 23.3 & 0 & - & 2 & $(4.0)$ \\
\hline $\mathrm{T} 12$ & $82(33 / 49)$ & 146.9 & \pm 33.2 & 146.2 & \pm 44.4 & 2 & $(6.1)$ & 1 & $(2.0)$ \\
\hline $\mathrm{T} 0-\mathrm{T} 12$ & $78(30 / 48)$ & -20.97 & \pm 46.62 & -14.02 & \pm 43.87 & & & & \\
\hline \multicolumn{10}{|c|}{ Zinc $(9.2-18.4 \mu \mathrm{mol} / \mathrm{L}(U S: 60-120 \mu \mathrm{g} / \mathrm{dL})$} \\
\hline T6 & $87(35 / 52)$ & 11.9 & \pm 1.65 & 12.1 & \pm 1.82 & 2 & $(5.7)$ & 1 & (1.9) \\
\hline $\mathrm{T} 12$ & $79(33 / 46)$ & 11.8 & \pm 2.0 & 11.7 & \pm 1.5 & 0 & - & 2 & $(4.3)$ \\
\hline T0-T12 & $75(31 / 44)$ & -0.22 & \pm 2.23 & -0.68 & \pm 2.27 & & & & \\
\hline
\end{tabular}

Data are presented as mean \pm standard deviation and counts (percentages)

Information about compliance was missing for 17 patients at $\mathrm{T} 6$ and 23 patients at $\mathrm{T} 12$. These patients were excluded from analysis for that time point. Non-users of multivitamin supplements were also excluded

US United States (units), $s M V S$ standard multivitamin supplement

$* p<0.05$

because they do not make a distinction between the different types of weight loss surgery.

\section{Vitamin D Deficiency}

Vitamin D deficiency was the most prevalent micronutrient deficiency at baseline (76.1\%). During the study, $10 \%$ of the patients was deficient. This is not in line with other studies, reporting between 16 and $89 \%$ of patients being deficient [7,
27, 28]. Next to supplementation and monitoring post-surgery, the improvement in vitamin D status is probably due to our preoperative supplementation protocol. According to the systematic review of Dix et al., only 3 of the 17 included studies used additional supplementation to improve vitamin D status before SG [27]. Prevalence rates of post-operative vitamin D deficiencies in studies using a preoperative treatment protocol range from 14 to $36 \%$ [9, 15, 17, 29], being closer to our observation. 
Calcium and magnesium deficiencies were rare during the first year post-sleeve, but low levels of phosphate were found more frequently. Hypophosphatemia is usually due to vitamin $\mathrm{D}$ deficiency [30]. However, because of the low prevalence of vitamin D deficiencies, we could not confirm this in the present study. Nevertheless, the dose of vitamin D in an SGspecific supplement should be increased to the levels advised for RYGB patients by the ASMBS ( $75 \mu \mathrm{g}$ per day) to also improve post-operative phosphate levels [25].

\section{Vitamin B1 Deficiency}

Vitamin B1 deficiencies are not commonly reported after SG, probably because they are not routinely measured. We found lowered vitamin B1 levels in nine patients $(7.3 \%)$ throughout the study period, but none showed clinical symptoms. This differs from RYGB patients in whom such deficiencies hardly occur $[7,8]$. Theoretically, this could be explained by the higher risk of minimized intake and vomiting after SG compared to a RYGB. When thiamin levels are below the adequate level, this can result in serious cardiovascular and neurologic consequences such as Wernicke's encephalopathy (WE) and beriberi [31, 32]. Risk factors known to cause post-bariatric WE include alcohol consumption, vomiting, and rapid weight loss, but also poor compliance with vitamin supplementation is an important predisposing factor [28, 32, 33].

For non-vomiting patients, the dose of thiamin required to prevent deficiencies post-sleeve should be increased from 2 to $\pm 3 \mathrm{mg}$. In our opinion, the recommended dose of $12 \mathrm{mg}$ per day by the ASMBS [25] is thus highly overestimated.

\section{Zinc Deficiency}

As with thiamin, only a few studies have evaluated zinc status after bariatric surgery, mainly focusing on one type of surgery (RYGB). In the present study, prevalence of zinc deficiency was $13 \%$, which is quite low compared to the wide range of 5$39 \%$ described in the available literature [12, 17, 34-36]. It is suggested that initially, zinc deficiency may be caused by malabsorption and protein malnutrition [35]. In our study, the only marker for protein status was albumin. About half of the patients that were deficient for zinc also had low albumin levels, and at 12 months, serum zinc levels were significantly correlated with serum albumin levels $(r=0.496)$. Other factors associated with zinc deficiency include a reduction of gastric $\mathrm{HCl}$ limiting zinc absorption, and inadequate intake of dietary zinc because of intolerance to foods rich in zinc such as red meat $[35,37]$.

The dose of $15 \mathrm{mg}$ of zinc in WLS Optimum 1.0 was not sufficient to prevent deficiencies. However, this dose was already higher than the recommended dose of 8-11 $\mathrm{mg}$ per day according to the ASMBS [25]. For SG patients, recommendations should be increased to at least the levels advised in RYGB patients (8-11 mg/day to $16-22 \mathrm{mg} /$ day).

\section{Hypervitaminosis}

Some patients showed excess serum levels of vitamin B1 (13\%) and vitamin B6 (27\%) throughout the study period. For both vitamins, excess cases were more prevalent than deficient ones. Complications of high doses of vitamin B1 are rare as the body can excrete excess amounts of thiamin in the urine $[13,31]$. However, excessive serum levels of vitamin B6 can cause neuropathic symptoms [38]. Despite the higher dose of vitamin B6 in WLS Optimum (143\% RDA) compared to the sMVS (100\% RDA), no difference in prevalence of hypervitaminosis was found between the two groups. In three patients, extremely high serum levels (>200 nmol/L) of vitamin B6 were found. Clinical manifestations of vitamin toxicity have not been actively investigated in the present study. Consequently, it is difficult to ascertain whether the observed excess levels are clinically relevant. High serum vitamin B6 levels can also occur due to over-use of vitamin supplements. As serum folic acid concentrations rapidly increase after intake [39], these concentrations can be used as a marker for compliance of MVS intake in countries where it is not a food additive. In our study, serum vitamin B6 levels were significantly correlated with serum folic acid levels at 12 months $(r=0.494)$, but it is unclear if patients indeed overdosed the MVS.

\section{Limitations}

The present study has some limitations, especially the relatively high percentage of incompliant patients which most likely led to underpowering. Even when provided free of charge, about one third of the patients were not compliant to the supplement protocol. With respect to product optimization, this finding is very important as it indicates that this version WLS Optimum was probably not well tolerated.

In addition, information on compliance was subjective (via questionnaires and medical files) and incomplete which might have led to an overestimation of compliant patients. Yet, comparing self-reported intake to blister counting in a previous study showed that the majority of the patients are honest in their self-reports. Besides, presuming that serum folic acid levels can serve as a marker for compliance, the absence of folic acid deficiencies in the per protocol analyses implies that these patients were indeed compliant.

Furthermore, only preoperative deficiencies for vitamin B12 and vitamin D were treated. Not correcting for all preoperative deficiencies could have affected our findings regarding the efficacy of both multivitamin supplements in relation to the observed nutritional status.

Despite these limitations, we believe that we can draw important conclusions about nutritional status of the investigated 
micronutrients after SG and the need for long-term nutritional follow-up and maintenance of routine multivitamin supplementation.

\section{Conclusion}

This randomized controlled study showed that nutritional deficiencies are prevalent after sleeve gastrectomy. Despite the fact that the investigated, specialized multivitamin supplement contained elevated doses of multiple vitamins and minerals, it only significantly affected serum levels of folic acid, PTH and vitamin B1, and anemia rates compared to a standard MVS. This indicates that there is a clear need to further optimize multivitamin supplementation for sleeve patients. These supplements should contain higher doses of elementary iron, folic acid, vitamin B12, vitamin D, vitamin B1, and zinc to prevent deficiencies post-operatively. However, caution is needed to prevent oversupplementation as we found that most of the recommended doses for WLS preventative supplements according to the ASMBS guideline might be overestimated.

Besides, non-compliance with multivitamin supplements was frequently encountered. More research is needed to identify which factors affect (non-)compliance and how this can be improved.

Acknowledgments We would like to thank L. Roovers for her statistical assistance.

Sources of Support For this study, FitForMe (Rotterdam, the Netherlands) provided WLS Optimum and placebo capsules for all 150 patients.

Authors' Contributions FJB and EOA designed the research; WS and NP conducted the research; LH analyzed data; LH, EOA, LND and EJH wrote the paper; EOA had primary responsibility for final content.

\section{Compliance with Ethical Standards}

Conflict of Interest FJB and EOA are consultants for FitForMe. All other authors have nothing to declare. WLS Optimum and 100\% RDA placebos were provided free of charge.

Ethical Approval All procedures performed in studies involving human participants were in accordance with the ethical standards of the institutional and national research committee and with the 1964 Helsinki declaration and its later amendments or comparable ethical standards.

Informed Consent Informed consent was obtained from all individual participants included in the study.

Open Access This article is distributed under the terms of the Creative Commons Attribution 4.0 International License (http:// creativecommons.org/licenses/by/4.0/), which permits unrestricted use, distribution, and reproduction in any medium, provided you give appropriate credit to the original author(s) and the source, provide a link to the Creative Commons license, and indicate if changes were made.

\section{References}

1. Nguyen NT, Nguyen B, Gebhart A, et al. Changes in the makeup of bariatric surgery: a national increase in use of laparoscopic sleeve gastrectomy. J Am Coll Surg. 2013;216(2):252-7. https://doi.org/ 10.1016/j.jamcollsurg.2012.10.003.

2. Higa K, Himpens J, Welbourn R, Dixon J, Kinsman R, Walton P. Third IFSO global registry report. Oxfordshire, United Kingdom: 2017.

3. Kehagias I, Zygomalas A, Karavias D, et al. Sleeve gastrectomy: have we finally found the holy grail of bariatric surgery? A review of the literature. Eur Rev Med Pharmacol Sci. 2016;20(23):4930 42.

4. Juodeikis Z, Brimas G. Long-term results after sleeve gastrectomy: a systematic review. Surg Obes Relat Dis Off J Am Soc Bariatric Surg. 2017;13(4):693-9. https://doi.org/10.1016/j.soard.2016.10. 006.

5. Lupoli R, Lembo E, Saldalamacchia G, et al. Bariatric surgery and long-term nutritional issues. World J Diabetes. 2017;8(11):464-74. https://doi.org/10.4239/wjd.v8.i11.464.

6. Weng TC, Chang $\mathrm{CH}$, Dong $\mathrm{YH}$, et al. Anaemia and related nutrient deficiencies after roux-en- $Y$ gastric bypass surgery: a systematic review and meta-analysis. BMJ Open. 2015;5(7):e006964. https:// doi.org/10.1136/bmjopen-2014-006964.

7. Ben-Porat T, Elazary R, Goldenshluger A, et al. Nutritional deficiencies four years after laparoscopic sleeve gastrectomy-are supplements required for a lifetime? Surg Obes Relat Dis Off J Am Soc Bariatric Surg. 2017;13(7):1138-44. https://doi.org/10.1016/j. soard.2017.02.021.

8. Ruiz-Tovar J, Llavero C, Zubiaga L, et al. Group O. maintenance of multivitamin supplements after sleeve Gastrectomy. Obes Surg. 2016;26(10):2324-30. https://doi.org/10.1007/s11695-016-20845.

9. Coupaye M, Riviere P, Breuil MC, et al. Comparison of nutritional status during the first year after sleeve gastrectomy and roux-en-Y gastric bypass. Obes Surg. 2014;24(2):276-83. https://doi.org/10. 1007/s11695-013-1089-6.

10. Guan B, Yang J, Chen Y, et al. Nutritional deficiencies in Chinese patients undergoing gastric bypass and sleeve Gastrectomy: prevalence and predictors. Obes Surg. 2018;28:2727-36. https://doi.org/ 10.1007/s11695-018-3225-9.

11. Kwon Y, Kim HJ, Lo Menzo E, et al. Anemia, iron and vitamin B12 deficiencies after sleeve gastrectomy compared to roux-en-Y gastric bypass: a meta-analysis. Surg Obes Relat Dis Off J Am Soc Bariatric Surg. 2014;10(4):589-97. https://doi.org/10.1016/j.soard. 2013.12.005.

12. Moize V, Andreu A, Flores L, et al. Long-term dietary intake and nutritional deficiencies following sleeve gastrectomy or roux-en-Y gastric bypass in a mediterranean population. J Acad Nutr Diet. 2013;113(3):400-10. https://doi.org/10.1016/j.jand.2012.11.013.

13. Aarts EO, Janssen IM, Berends FJ. The gastric sleeve: losing weight as fast as micronutrients? Obes Surg. 2011;21(2):207-11. https://doi.org/10.1007/s11695-010-0316-7.

14. Al-Mutawa A, Al-Sabah S, Anderson AK, et al. Evaluation of nutritional status post laparoscopic sleeve Gastrectomy - 5-year outcomes. Obes Surg. 2017;28(6):1473-83. https://doi.org/10. 1007/s11695-017-3041-7.

15. Caron M, Hould FS, Lescelleur O, et al. Long-term nutritional impact of sleeve gastrectomy. Surg Obes Relat Dis. 2017;13(10): 1664-73. https://doi.org/10.1016/j.soard.2017.07.019.

16. Kikkas EM, Sillakivi T, Suumann J, et al. Five-year outcome of laparoscopic sleeve Gastrectomy, resolution of comorbidities, and risk for cumulative nutritional deficiencies. Scand J Surg. $2018 ; 1457496918783723 \mathrm{https} / / /$ doi.org/10.1177/ 1457496918783723. 
17. van Rutte PW, Aarts EO, Smulders JF, et al. Nutrient deficiencies before and after sleeve gastrectomy. Obes Surg. 2014;24(10):163946. https://doi.org/10.1007/s11695-014-1225-y.

18. Parikh M, Issa R, McCrillis A, et al. Surgical strategies that may decrease leak after laparoscopic sleeve gastrectomy: a systematic review and meta-analysis of 9991 cases. Ann Surg. 2013;257(2): 231-7. https://doi.org/10.1097/SLA.0b013e31826cc714.

19. Yuval JB, Mintz Y, Cohen MJ, et al. The effects of bougie caliber on leaks and excess weight loss following laparoscopic sleeve gastrectomy. Is there an ideal bougie size? Obes Surg. 2013;23(10):168591. https://doi.org/10.1007/s11695-013-1047-3.

20. Dean AG, Sullivan KM, Soe MM. OpenEpi Version 3.0.1: Open Source Epidemiologic Statistics for Public Health [updated Apr 6, 2013]. Available from: www.OpenEpi.com

21. Steenackers N, Van der Schueren B, Mertens A, et al. Iron deficiency after bariatric surgery: what is the real problem? Proc Nutr Soc. 2018;77(4):445-55. https://doi.org/10.1017/S0029665118000149.

22. Hakeam HA, O'Regan PJ, Salem AM, et al. Impact of laparoscopic sleeve gastrectomy on iron indices: 1 year follow-up. Obes Surg. 2009;19(11):1491-6. https://doi.org/10.1007/s11695-009-9919-2.

23. Al-Mulhim AS. Laparoscopic sleeve Gastrectomy and nutrient deficiencies: a prospective study. Surg Laparosc Endosc Percutaneous Tech. 2016;26(3):208-11. https://doi.org/10.1097/SLE. 0000000000000270 .

24. Sioka E, Tzovaras G, Perivoliotis K, et al. Impact of laparoscopic sleeve Gastrectomy on gastrointestinal motility. Gastroenterol Res Pract. 2018;2018:4135813. https://doi.org/10.1155/2018/4135813.

25. Parrott J, Frank L, Rabena R, et al. American Society for Metabolic and Bariatric Surgery Integrated Health Nutritional Guidelines for the surgical weight loss patient 2016 update: micronutrients. Surg Obes Relat Dis. 2017;13(5):727-41. https://doi.org/10.1016/j. soard.2016.12.018.

26. Lam JR, Schneider JL, Zhao W, et al. Proton pump inhibitor and histamine 2 receptor antagonist use and vitamin B12 deficiency. JAMA. 2013;310(22):2435-42. https://doi.org/10.1001/jama. 2013.280490.

27. Dix CF, Bauer JD, Wright OR. A systematic review: vitamin D status and sleeve Gastrectomy. Obes Surg. 2017;27(1):215-25. https://doi.org/10.1007/s11695-016-2436-1.

28. Pellitero S, Martinez E, Puig R, et al. Evaluation of vitamin and trace element requirements after sleeve Gastrectomy at long term. Obes Surg. 2017;27(7):1674-82. https://doi.org/10.1007/s11695017-2557-1.

29. Zarshenas N, Nacher M, Loi KW, et al. Investigating nutritional deficiencies in a Group of Patients 3 years post laparoscopic sleeve
Gastrectomy. Obes Surg. 2016;26(12):2936-43. https://doi.org/10. 1007/s11695-016-2211-3

30. Mechanick JI, Youdim A, Jones DB, et al. Clinical practice guidelines for the perioperative nutritional, metabolic, and nonsurgical support of the bariatric surgery patient-2013 update: cosponsored by American Association of Clinical Endocrinologists, the Obesity Society, and American Society for Metabolic \& bariatric surgery. Obesity. 2013;21(Suppl 1):S1-27. https://doi.org/10.1002/oby. 20461

31. Kerns JC, Gutierrez JL. Thiamin. Adv Nutr. 2017;8(2):395-7. https://doi.org/10.3945/an.116.013979.

32. Schimpke S, Guerron AD. Prevalence and predictors of postoperative thiamine deficiency after vertical sleeve gastrectomy. Surg Obes Relat Dis. 2018;14(7):950-1. https://doi.org/10.1016/j.soard. 2018.04.014.

33. Milone M, Velotti N, Musella M. Wernicke encephalopathy following laparoscopic sleeve Gastrectomy-a call to evaluate thiamine deficiencies after restrictive bariatric procedures. Obes Surg. 2018;28(3):852-3. https://doi.org/10.1007/s11695-017-3083-X.

34. Gehrer S, Kern B, Peters T, et al. Fewer nutrient deficiencies after laparoscopic sleeve gastrectomy (LSG) than after laparoscopic roux-Y-gastric bypass (LRYGB)-a prospective study. Obes Surg. 2010;20(4):447-53. https://doi.org/10.1007/s11695-009-0068-4.

35. Salle A, Demarsy D, Poirier AL, et al. Zinc deficiency: a frequent and underestimated complication after bariatric surgery. Obes Surg. 2010;20(12):1660-70. https://doi.org/10.1007/s11695-010-0237-5.

36. Saif T, Strain GW, Dakin G, et al. Evaluation of nutrient status after laparoscopic sleeve gastrectomy 1,3, and 5 years after surgery. Surg Obes Relat Dis Off J Am Soc Bariatric Surg. 2012;8(5):542-7. https://doi.org/10.1016/j.soard.2012.01.013.

37. Livingstone C. Zinc: physiology, deficiency, and parenteral nutrition. Nutr Clin Pract Off Publ Am Soc Parenter Enter Nutr. 2015;30(3):371-82. https://doi.org/10.1177/0884533615570376.

38. Alsabah A, Al Sabah S, Al-Sabah S, et al. Investigating factors involved in post laparoscopic sleeve Gastrectomy (LSG) neuropathy. Obes Surg. 2017;27(5):1271-6. https://doi.org/10.1007/ s11695-016-2466-8.

39. Navarro M, Wood RJ. Plasma changes in micronutrients following a multivitamin and mineral supplement in healthy adults. J Am Coll Nutr. 2003;22(2):124-32. https://doi.org/10.1080/07315724.2003. 10719285.

Publisher's Note Springer Nature remains neutral with regard to jurisdictional claims in published maps and institutional affiliations. 